\title{
Comparison Research of Capability of Several Edge Detection Operators
}

\author{
Cui WANG, Yeli LI \& Yali QI \\ Beijing Key Laboratory of Signal and Information Processing for High-end Printing Equipments, Beijing \\ Institute of Graphic Communication, Beijing, China
}

\begin{abstract}
Edge detection is an important link of image segmentation and object extraction. This paper examines the five kinds of edge detection operators commonly used, uses $\mathrm{VC}++$ programming language to realize them, compares their test results and analysis their characteristics. It has a great reference value for learning edge detection and specific engineering application.
\end{abstract}

KEYWORD: Image segmentation; Edge detection; Detection operator

\section{INTRODUCTION}

Edge is the basics feature of images. Edges refer to the collection of pixels whose surrounding pixels have a step change or roof change. Edges exist in between the object and the background, object and object, primitive and primitive, so they are important characteristic of image segmentation[1]. Poggio pointed out that "edges may correspond to objects in the image(border), or may not, but they have very satisfactory natures that they can not only reduce the information to be processed greatly but also keep the shape information of objects in the image"[2]. Image segmentation based on geometry can reflect the object in the image more integrally than which based on color information[3]. Edge detection plays an important role in image recognition and computer analysis. Therefore, image edge detection has a vitally important research value and application prospect.

\section{DETECTION OPERATORS}

The edges of the object are reflected by the gray discontinuity. Firstly, edge detection detects the gray discontinuity, and then connects the discontinuous edge pixels into complete borders, which are the edges of objects. Edge detection operators examine the gray changes in a certain field of each pixel.

This article mainly discuss several kinds of commonly used edge detection operators, such as Roberts operator, Sobel operator, Prewitt operator, Kirsch operator and Canny operator.

\subsection{Roberts operator}

Roberts edge detection operator is according to the principle that any pair of differences which are perpendicular to each other can be used to calculate gradient, and the difference between the adjacent pixels in the diagonal direction approximates the edges[4]. The operator is given by formula (1).

$\left\{\begin{array}{l}\Delta_{x} f(x, y)=f(x, y)-f(x-1, y-1) \\ \Delta_{y} f(x, y)=f(x-1, y)-f(x, y-1)\end{array}\right.$

Gray amplitude is $G(x, y)=\sqrt{\Delta_{x} f^{2}+\Delta_{y} f^{2}}$

$G(x, y)$ is the gray value of the point $(x, y)$ after processing. $f(x, y)$ is the gray value of points before processing.

The template of Roberts operator is $2 \times 2$, all of points in the image are made convolutions from the two nuclear.

$$
\left[\begin{array}{cc}
1 & 0 \\
1 & -1
\end{array}\right]\left[\begin{array}{cc}
0 & 1 \\
-1 & 0
\end{array}\right]
$$

\subsection{Sobel operator}

Sobel operator uses the principle that the gray weighted algorithm of the up, down, left and right neighborhoods attends extremum at edge points to detect edges[5]. Taking a weighted average of the first, then differential, calculating gradient finally. The calculation method of the operator is given by formula (2). 


$$
\left\{\begin{aligned}
\Delta_{x} f(x, y)= & {[f(x-1, y+1)+2 f(x, y+1)+f(x+1, y+1)] } \\
- & {[f(x-1, y-1)+2 f(x, y-1)+f(x+1, y-1)] } \\
\Delta_{y} f(x, y)= & {[f(x-1, y-1)+2 f(x-1, y)+f(x-1, y+1)] } \\
& -[f(x+1, y-1)+2 f(x+1, y)+f(x+1, y+1)]
\end{aligned}\right.
$$

Gray amplitude is $G(x, y)=\left|\Delta_{x} f\right|-\left|\Delta_{y} f\right|$

The following two convolution kernels form the Sobel edge detection operator, the former has the greatest influence on horizontal edges and the latter has the biggest influence on vertical edges. In practial application, each pixel in the image takes a maximum of the two convolution kernels as the output. The result is a gradient magnitude image.

$$
\left[\begin{array}{ccc}
-1 & -2 & -1 \\
0 & 0 & 0 \\
1 & 2 & 1
\end{array}\right]\left[\begin{array}{ccc}
-1 & 0 & 1 \\
-2 & 0 & 2 \\
-1 & 0 & 1
\end{array}\right]
$$

\subsection{Prewitt operator}

The method of Prewitt operator is similar to Sobel operator, using the gray weighted algorithm of the up, down, left and right neighborhoods attends extremum at edge points to detect edges[4]. The following two convolution kernels form the Prewitt edge detection operator. All of points in the image are made convolutions from the two nuclears and select the maximum as output.

$$
\left[\begin{array}{ccc}
-1 & -1 & -1 \\
0 & 0 & 0 \\
1 & 1 & 1
\end{array}\right] \quad\left[\begin{array}{ccc}
1 & 0 & -1 \\
1 & 0 & -1 \\
1 & 0 & -1
\end{array}\right]
$$

\subsection{Kirsch operator}

Kirsch operator consists of the following eight convolution kernels. All of points in the image are made convolutions from the eight nuclears that each nuclear makes the maximum response to a particular edge direction. The maximum of all average differences in eight directions is the gradient magnitude image [6].

$\left.\begin{array}{lcc}{\left[\begin{array}{ccc}5 & 5 & 5 \\ -3 & 0 & -3 \\ -3 & -3 & -3\end{array}\right]} & {\left[\begin{array}{ccc}-3 & 5 & 5 \\ -3 & 0 & 5 \\ -3 & -3 & -3\end{array}\right]} & {\left[\begin{array}{ccc}-3 & -3 & 5 \\ -3 & 0 & 5 \\ -3 & -3 & 5\end{array}\right]}\end{array}\right]\left[\begin{array}{ccc}-3 & -3 & -3 \\ -3 & 0 & 5 \\ -3 & 5 & 5\end{array}\right]$

\subsection{Canny edge detection}

The steps of Canny edge detection as follows:

1) smoothing image with Gaussian filter

Canny algorithm uses the two-dimensional Gaussian function to smooth the image firstly:
$G(x, y)=\frac{1}{2 \pi \sigma^{2}} \exp \left(-\frac{x^{2}+y^{2}}{2 \sigma^{2}}\right)$

$\sigma$ is the parameter of Gaussian filter and controls the degree of smooth. The filter with a smaller $\sigma$ has a high positoning accuracy but a low signal-to-noise ratio; the filter with a bigger $\sigma$ is just the opposite. So we should select the Gaussian filter parameter according to the need.

2) the calculation of gradient magnitude and gradient direction

Traditional Canny algorithm uses the first order differential operator to calculate the gradient amplitude and gradient direction of each point in the image after smooth for the gradient amplitude image $G$ and the gradient direction image $\theta$. The partial derivatives of the point $(i, j)$ in two directions are:

$$
\begin{aligned}
& G_{x}(i, j)=(I(i, j+1)-I(i, j)+I(i+1, j+1)-I(i+1, j)) / 2 \\
& G_{y}(i, j)=(I(i, j)-I(i+1, j)+I(i, j+1)-I(i+1, j+1)) / 2
\end{aligned}
$$

So the gradient magnitude and gradient direction of point $(i, j)$ are:

$$
\begin{aligned}
& G(i, j)=\sqrt{G_{x}^{2}(i, j)+G^{2}(i, j)} \\
& \theta(i, j)=\arctan \left(\frac{G_{x}(i, j)}{G_{y}(i, j)}\right)
\end{aligned}
$$

3) the non-maxima suppression to the gradient magnitude

The non-maxima suppression (NMS) is by refining roofs in the gradient magnitude image $G$ and keeping local maximum of the amplitude only, in order to locate edges accurately. Canny algorithm interpolates within the $3 \times 3$ neighborhood centered on the point $(i, j)$ along the gradient direction $\theta(i, j)$ in the image $G$.If the gradient amplitude $G(i, j)$ of the point $(i, j)$ is bigger than the adjacent two interpolation along the direction $\theta(i, j)$, point $(i, j)$ would be marked as the candidate edge pixels, otherwise is marked as non-edge pixels ${ }^{[7]}$. After that, we can get the candidate edge image $N$. The non-maxima suppression result is shown in figure 1 .

The color and numerical value of each block in figure 1 represent the value of gradients. The direction of arrows represent the direction of gradients. The blocks with white edges represent the edge points after the non-maxima suppression. 


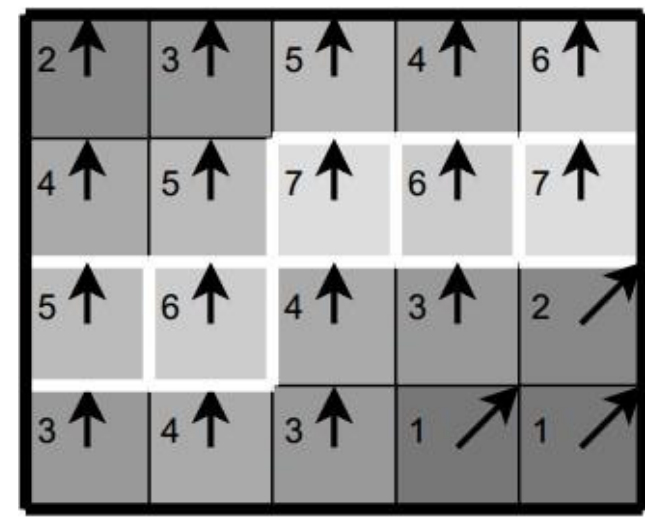

Figure1 the effect picture of non-maxima suppression

4) edge tracking with double threshold algorithm

Canny algorithm detects and links up the final edge from candidate points by double threshold algorithm. Firstly, we should select high threshold $T_{h}$ and low threshold $T_{l}$. Then detect either pixel $(i, j)$ marked as candidate points in the picture $N$. If the gradient amplitude $G(i, j)$ of $\operatorname{pixel}(i, j)$ is bigger than $T_{h}$, this point must be the edge point. If it is smaller than $T_{l}$, this point must not be the edge point. If it is between $T_{h}$ and $T_{l}$, we will base on the connectivity of edges for further judgment. If there are edge pixels in the areas of it, it is a edge point, otherwise it is a non-edge pixel ${ }^{[7]}$.

\section{THE EXPERIMENTAL RESULTS AND COMPARATIVE ANALYSIS}

This paper uses $\mathrm{VC}++$ programming language to detect the above five kinds of edge detection operators. Firstly, the original image is preprocessed, then uses Roberts operator, Sobel operator, Prewitt operator, Kirsch operator and Canny operator to detect edges respectively. Through the comparison of experimental results, we can know the characteristics of all of the operators. The original image is shown in figure 2(a) and the edge detection results are shown in figure 2(b) to 2(f). As shown in figures, Roberts operator is well-position but detects fewer details about edges; the edge detection effect of Sobel is similar to Prewitt, outline is clear but edges are wide; the edges which are detected by Kirsch operator are fuzzy and the positioning accuracy is low; Canny operator uses Gaussian function to smooth the image and detects edges later. The detected edges are more complete, the connecting is better, the positioning accuracy is higher.

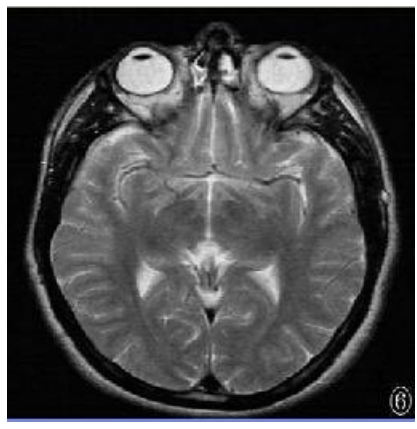

(a) the original image

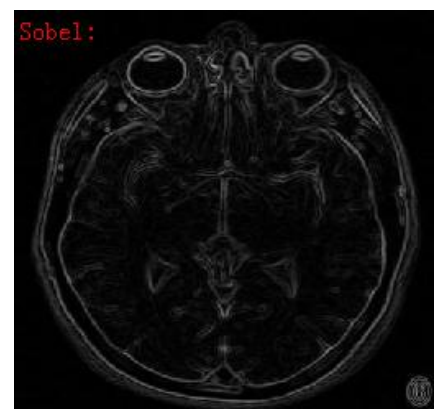

(c)the Sobel effect

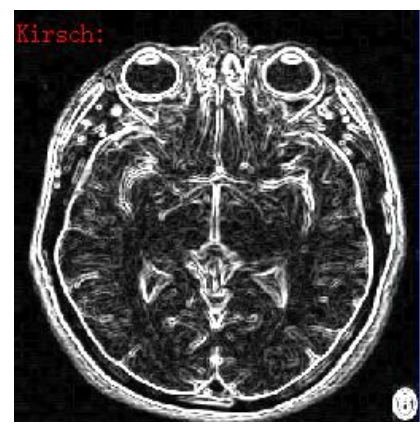

(e)the Kirsch effect

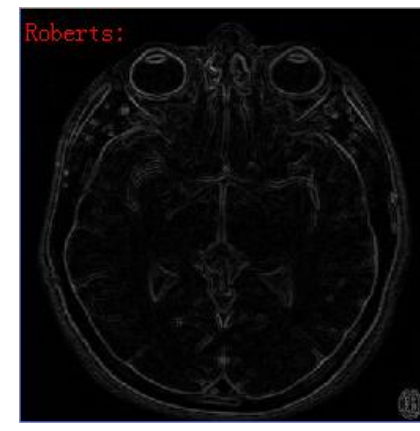

(b) the Roberts effect

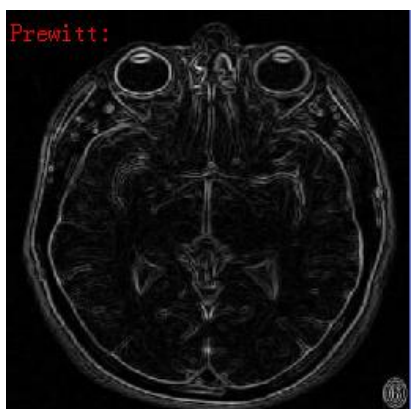

(d) the Prewitt effect

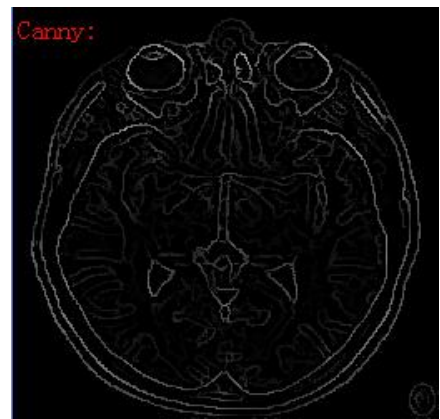

(f) the Canny effect
Figure2 the edge detection effect comparison of several operators

\section{CONCLUSION}

Edge detection has been a hot research topic in image processing and there are many different methods to realize. This paper gives the contrast analysis of several commonly used edge detection operators which provides the certain basis for selecting suitable operators according to different needs.

\section{ACKNOWLEDGEMENTS}

The authors would like to thank Supported by the Education Commission of Beijing (TJSHG201310015016), Project funded by the BIGC Key Project (E-A-2014-11), Program funded by the Beijing outstanding talents cultivation project (2013D002048000003). 


\section{REFERENCES}

[1] Weibo WEI, Xiaoting RUI. Study on edge detection method. Computer Engineering and Applications, 2006, $42(30): 88-91$.

[2] T Poggio, H Voorhees, A Yuille. A Regularized Solution to Edge Detection. Journal of Complexity, 1988, 2(4):106123.

[3] Shengxiao NIU, Sheng WANG, Jingjing YANG, Gengsheng CHEN. A Fast Image Segmentation Algorithm Fully Based on Edge Information. Journal of ComputerAided Design \& Computer Graphics, 2012, 24(11):14101419.
[4] Wennuan OU. Analysis and Comparison of Several Common Edge Detection Operators. Modern Computer, 2010.05:75-77.

[5] Shangju HU, Guofa TIAN, Jiangbo SHEN.The analysis and comparison of edge detection operators. 2008.09:4849.

[6] Yuyin TAN, Qi CHEN. The contrast analysis of several edge detection operators. 2010.29:8326-8327.

[7] John Canny, Member, IEEE. A Computational Approach to Edge Detection. IEEE Trans Pattern Analysis and Machine Intelligence, 1986, 8(6): 679-698. 